\title{
On the nature of large-scale mixing in the stratosphere and mesosphere
}

Article

Published Version

Shepherd, T. G., Koshyk, J. N. and Ngan, K. (2000) On the nature of large-scale mixing in the stratosphere and mesosphere. Journal of Geophysical Research, 105 (D10). pp. 12433-12446. ISSN 0148-0227 doi: https://doi.org/10.1029/2000JD900133 Available at https://centaur.reading.ac.uk/32852/

It is advisable to refer to the publisher's version if you intend to cite from the work. See Guidance on citing.

Published version at: http://dx.doi.org/10.1029/2000JD900133

To link to this article DOI: http://dx.doi.org/10.1029/2000JD900133

Publisher: American Geophysical Union

All outputs in CentAUR are protected by Intellectual Property Rights law, including copyright law. Copyright and IPR is retained by the creators or other copyright holders. Terms and conditions for use of this material are defined in the End User Agreement.

\section{www.reading.ac.uk/centaur}

\section{CentAUR}

Central Archive at the University of Reading 
Reading's research outputs online 


\title{
On the nature of large-scale mixing in the stratosphere and mesosphere
}

\author{
Theodore G. Shepherd and John N. Koshyk \\ Department of Physics, University of Toronto, Toronto, Ontario, Canada \\ Keith Ngan ${ }^{1}$ \\ Department of Geophysical Sciences, University of Chicago, Chicago, Illinois
}

\begin{abstract}
Studies of tracer transport in the stratosphere have shown that adiabatic quasi-horizontal tracer evolution is controlled primarily by the large-scale low-frequency component of the flow. This behavior is consistent with the concept of chaotic advection, wherein the Eulerian velocity field is spatially coherent and temporally quasi-regular on timescales over which the Lagrangian evolution is chaotic. In this study, winds from a middle atmosphere general circulation model (the Canadian Middle Atmosphere Model) are used to compare and contrast the nature of tracer evolution in the stratosphere and mesosphere. It is found that the concept of chaotic advection is relevant in the stratosphere but not in the mesosphere. The explanation for this behavior is the increased strength of gravity wave activity in the mesosphere as compared with the stratosphere, which leads to shallower kinetic energy spectra on synoptic scales and a much shorter Eulerian correlation time. The shallower kinetic energy spectra imply that tracer evolution in the mesosphere is spectrally local, in contrast with the spectrally nonlocal regime that prevails in the stratosphere. This means that tracer advection calculations in the mesosphere are controlled primarily by the gravity wave spectrum and are intrinsically resolution dependent.
\end{abstract}

\section{Introduction}

Many studies have shown that Lagrangian motion in the extratropical wintertime stratosphere is, over adiabatic timescales (i.e., up to 2 weeks or so), determined almost entirely by the large-scale balanced wind field [e.g., Norton, 1994; Waugh and Plumb, 1994]. It is this feature that accounts for the remarkable success of highresolution contour and particle advection calculations that use coarse-grained analyzed winds [Waugh et al., 1994; Newman and Schoeberl, 1995]. The same feature also explains the formation of filaments and laminae, as seen in in situ measurements of long-lived tracers [Strahan and Mahlman, 1994; Orsolini, 1995; Gibson-Wilde. et al., 1997], provides the most plausible explanation of the approximately inverse square power law scaling seen in spatial wavenumber spectra of $\mathrm{N}_{2} \mathrm{O}$ and $\mathrm{O}_{3}[\mathrm{Ngan}$ and Shepherd, 1997], and is the basis for a theory of mix-down times in the stratosphere [Thuburn and Tan, 1997].

\footnotetext{
${ }^{1}$ Now at Department of Physics, University of Toronto, Toronto, Ontario, Canada.

Copyright 2000 by the American Geophysical Union.

Paper number 2000JD900133.

0148-0227/00/2000JD900133\$09.00
}

The temporal and spatial coherence of the wind field in the extratropical wintertime stratosphere reflects the dominant role of breaking planetary-scale waves [ $\mathrm{McIn-}$ tyre and Palmer, 1983], which provide an "organizing structure" for transport and mixing [Ngan and Shepherd, 1999a]. These features represent the defining characteristics of "chaotic advection." In its strictest definition, chaotic advection refers to a situation in which the Lagrangian evolution is chaotic while the wind field (as seen in the Eulerian frame) is temporally periodic, with smooth spatial structure. However, this definition is unnecessarily restrictive, for the concept extends naturally to a situation in which the wind field is temporally quasi-regular, with an Eulerian correlation time that is long in comparison with the Lagrangian correlation time [Swanson and Pierrehumbert, 1997]. By computing the correlation times of winds from an idealized shallow water model, as well as from a comprehensive general circulation model, Ngan and Shepherd [1999a, b] have argued that this situation applies to the extratropical wintertime stratosphere.

However, the extratropical wintertime stratosphere is a rather special place. Charney-Drazin filtering [e.g., Andrews et al., 1987] eliminates all but the planetaryscale Rossby waves, while gravity wave amplitudes generally remain weak. This leaves a wind field that is very 
strongly band limited in wavenumber and frequency, precisely what is required for chaotic advection.

In the mesosphere, by contrast, upward propagating planetary waves are weaker, having largely been attenuated through breaking in the stratospheric "surf zone," while gravity wave amplitudes can be expected to have grown substantially (because of the decreasing air density). This leads to a wind field that is quite different in character from that in the stratosphere. Global measurements of mesospheric winds do not exist with enough temporal and spatial resolution to adequately characterize the flow, but ground-based single-station measurements suggest strong gravity wave activity $[\mathrm{Bal}$ sley and Carter, 1982; Wilson et al., 1991; Whiteway and Carswell, 1995] (note that the latter two references are based on temperature measurements). In the absence of adequate measurements, an alternative is to examine the wind field from a comprehensive middle atmosphere general circulation model (MAGCM). Although the model fields may not be completely realistic, they ought to be at least qualitatively correct in their spatio-temporal characteristics and thus provide a plausible surrogate atmosphere.

Koshyk et al. [1999a] have recently compared the spatial wavenumber kinetic energy spectra from five different MAGCMs. Despite quantitative differences between the models, a number of qualitative features are common to them all. The model stratospheres are characterized by steep spectra with a strong energy peak at large scales, the flow being largely balanced (i.e., the rotational component dominates the divergent component). As the altitude increases, the unbalanced component increases in amplitude much more rapidly than does the balanced component. The energy spectra of the model mesospheres exhibit a shallow tail, the flow being largely unbalanced at the smaller scales (i.e., there is approximate equipartition between the divergent and rotational components). Detailed analyses of the Geophysical Fluid Dynamics Laboratory (GFDL) SKYHI model confirm that gravity waves do account for a significant component of the small-scale flow [Hayashi et al., 1989]. Consistent with vertical propagation, the small-scale variability in the middle atmosphere is generated primarily by energy transfer from below rather than by a cascade from the larger horizontal scales (J. N. Koshyk and K. Hamilton, The horizontal kinetic energy spectrum and spectral budget simulated by a high-resolution troposphere-stratosphere-mesosphere GCM, submitted to Journal of the Atmospheric Sciences).

The change in slope of the kinetic energy spectrum referred to above has important implications for tracer evolution. Bennett [1984] identified two distinct dynamical regimes: "spectrally local," where tracer evolution at a given length scale is controlled by the velocity field at the same length scale, and "spectrally nonlocal," where tracer evolution is controlled by the velocity field at much larger length scales (essentially, the energy-containing scales). Bennett [1984] showed that for a kinetic energy spectrum of the form $E(k) \propto k^{-\alpha}$, where $k$ is the horizontal wavenumber, the marginal exponent is $\alpha=3$. This can be seen most easily from the analysis of Babiano et al. [1985], who argued that the characteristic timescale $\tau$ at wavenumber $k$ is given by

$$
\tau(k)=\left\{\int_{0}^{k} p^{2} E(p) d p\right\}^{-1 / 2} .
$$

This expression can be understood by noting that the relative dispersion of two fluid elements is determined by the structure function, which depends on the horizontal derivative of the velocity field at scales larger than the scale in question. The integral in (1) is essentially independent of its upper limit if $\alpha>3$ (the nonlocal case) but is controlled by its upper limit if $\alpha<3$ (the local case). It follows that in the nonlocal case, a single timescale set by the large-scale flow controls the tracer evolution; in the local case, $\tau(k)$ does not approach a constant as $k \rightarrow \infty$ and the characteristic timescale decreases as the horizontal scale decreases. Bartello [2000] has noted the implications of these two regimes for the representation of fine-scale tracer structure from low-resolution winds, in the context of the enstrophy- and energy-cascading inertial subranges of two-dimensional turbulence. Clearly, chaotic advection is a special case of nonlocal dynamics and is inconsistent with local dynamics.

The results of Koshyk et al. [1999a] suggest that the stratosphere, with a kinetic energy spectrum steeper than $k^{-3}$, obeys nonlocal dynamics, while the mesosphere, with a spectrum shallower than $k^{-3}$, obeys local dynamics. It follows that the nature of adiabatic tracer evolution should be quite different in these two regions of the middle atmosphere. But quite apart from the increased spatial irregularity of the velocity field in the mesosphere (reflected in the shallower kinetic energy spectrum), one also expects increased temporal irregularity due to the gravity wave activity, something that is not captured in the simple arguments leading to (1).

In this study, we examine the implications of this increased gravity wave activity (or more precisely, the associated increased spatial and temporal irregularity of the velocity field) for adiabatic Lagrangian motion in the mesosphere. Using winds from the Canadian Middle Atmosphere Model (CMAM), off-line advection calculations are performed and various diagnostics (Lagrangian and Eulerian correlation times, probability distribution functions (PDFs) of Liapunov exponents) considered. Spatial and temporal truncations of the velocity field are used to determine the extent to which the tracer evolution is spectrally local or nonlocal and the role of temporal coherence. We find that the concept of chaotic advection is not applicable in the model mesosphere because the winds are too irregular, temporally and spatially. It follows that most of our intuition regarding transport and mixing in the stratosphere is probably inapplicable to the mesosphere. 
In the vicinity of the extratropical tropopause, around 200 mbar, the concept of chaotic advection also bears further scrutiny. Here the issue is not gravity waves, but rather the fact that Charney-Drazin filtering has not removed the synoptic-scale motions; thus the balanced flow is not strongly band limited in wavenumber. Indeed, kinetic energy spectra at these levels follow an approximately $k^{-3}$ power law scaling, which is consistent with two-dimensional turbulence theory [Boer and Shepherd, 1983]; according to Bennett [1984] and Babiano et al. [1985], this corresponds to a weakly nonlocal regime (the integral in (1) diverges logarithmically as $k \rightarrow \infty)$. We find that chaotic advection is of uncertain relevance in this region, although the breakdown is much less scvere than that in the mesosphere.

\section{Methodology}

Ideally, one would like to perform transport studies using observed winds. However, global wind observations of sufficient resolution in space and time are not available in the mesosphere. Even in the stratosphere, where analyzed winds exist, the gravity wave component is generally filtered from the observations. Thus our approach is to use a MAGCM as a surrogate atmosphere.

The CMAM is a comprehensive MAGCM based on the primitive equations of motion; the version used here is documented by Beagley et al. [1997]. The model extends from the surface of the Earth to approximately $95 \mathrm{~km}$. It has a spectral formulation in the horizontal with a resolution of T32 (triangular truncation at spherical harmonic index $n=32$ ) and a finite difference formulation in the vertical with a resolution of approximately $3 \mathrm{~km}$ throughout the middle atmosphere. (The vertical resolution in the lowermost stratosphere and troposphere is much higher, with 25 levels below 25 $\mathrm{km}$.) The hybrid vertical coordinate is terrain-following at low levels and essentially coincident with pressure in the middle atmosphere. The time-stepping scheme is semi-implicit, with a time step of $5 \mathrm{~min}$. The model winds are transformed to a spatial Gaussian grid with $32 \times 64$ latitude-longitude grid points, interpolated to isentropic surfaces and archived every 3 hours.

CMAM, like any primitive equations model, explicitly simulates (hydrostatic) internal gravity waves whenever they are forced by mechanisms internal to the model that project on the time and space scales resolved by the model. It is the role of these waves (or more generally, of the spectrum of unbalanced motion) that is the focus of this study. Resolved gravity waves propagate upward and dissipate through a variety of mechanisms: radiative damping, horizontal and vertical diffusion, and a Rayleigh drag sponge layer in the upper part of the model domain, above $80 \mathrm{~km}$. Since the divergent energy per unit mass in CMAM grows approximately like $\exp (z / H)$, where $H$ is the density scale height, from the tropopause up to the sponge layer
[Koshyk et al., 1999a, Figure 6], these dissipative processes do not appear to play a significant role in establishing the nature of the gravity wave spectrum below the sponge layer. (It is important to distinguish between resolved and parameterized gravity waves. This version of CMAM also includes a parameterization of subgrid-scale orographic gravity wave drag, but the effects of subgrid-scale gravity waves on transport are not represented here.)

For the purpose of this study we have chosen to focus on a single January from a long model simulation. The Lagrangian calculations are performed off-line by using horizontal winds on isentropic surfaces. Thus the calculations are completely adiabatic. Although real tracer motion will have a diabatic component, we are interested in isolating the adiabatic quasi-horizontal component since this dominates the velocity field. (This is true even for mesospheric gravity waves, which are believed to be largely hydrostatic and have radiative damping times of several days [Andrews et al., 1987].)

The contour advection code [Norton, 1994] is derived from a contour dynamics algorithm [Dritschel, 1989] and uses a fourth-order Runge-Kutta scheme for the time integration. For the calculations presented here, a 5 -min integration time step is used, although the results are fairly insensitive to this choice. Values of the winds at each time step are determined by linear interpolation in space and time between archived values. The particle advection (and Liapunov exponent) calculations are similar to those described by Ngan and Shepherd [1999a, b] and likewise use fourth-order Runge-Kutta integration with linearly interpolated wind fields, but with a time step of $225 \mathrm{~s}$. The computations are performed in two-dimensional (latitude-longitude) coordinates except north of $75^{\circ} \mathrm{N}$, where, to preserve accuracy, they are performed in three-dimensional Cartesian coordinates. The Liapunov exponents are computed using a matrix-based QR method [Geist et al., 1990; von Bremen et al., 1997].

\section{Results}

Figure 1 shows the results of contour advection calculations after 3 days on the $1000 \mathrm{~K}$ isentropic surface (approximately $35-\mathrm{km}$ altitude), which is chosen to be representative of the middle stratosphere. Figure 1a shows the result obtained using the full wind field interpolated between samples archived every 3 hours. The contours exhibit the smooth structure indicative of temporally persistent strain and characteristic of contour advection calculations using analyzed winds in the lower stratosphere [e.g., Waugh et al., 1994; Schoeberl and Newman, 1995]. There is a planetary wave breaking event, much as that of McIntyre and Palmer [1983], laminar wrapping of contours within the polar vortex, and some evidence of secondary rollup just south of Japan. The other three panels show the results obtained by using various subsets of the wind data (see caption for de- 

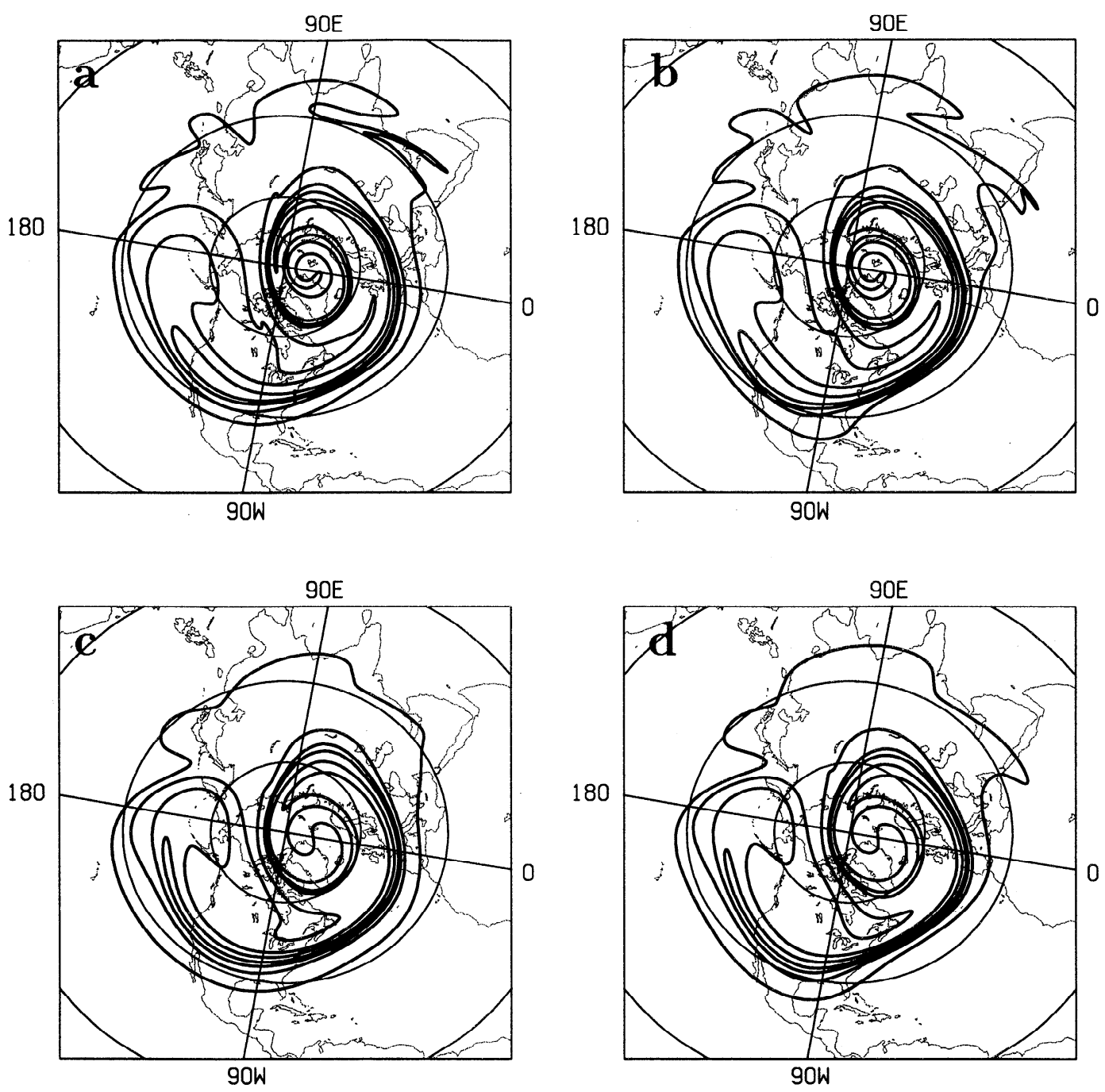

Figure 1. Distribution of four contours on the $1000 \mathrm{~K}$ isentropic surface after a 3-day integration period. The contours coincide initially with the $30^{\circ} \mathrm{N}, 45^{\circ} \mathrm{N}, 60^{\circ} \mathrm{N}$, and $75^{\circ} \mathrm{N}$ latitude circles. Contour advection with surgery is performed using January winds from a T32 version of the Canadian Middle Atmosphere Model with (a) winds sampled every 3 hours, (b) winds sampled every 18 hours, (c) winds truncated to T10 and sampled every 3 hours, and (d) winds truncated to T10 and sampled every 18 hours. Thin circles represent the equator, $30^{\circ} \mathrm{N}$, and $60^{\circ} \mathrm{N}$.

tails). The results are rather insensitive to reduction of the temporal or spatial resolution: even a truncation to T10 winds and 18-hour sampling (Figure 1d) gives little change. Thus the contour evolution is determined almost entirely by the large-scale low-frequency component of the flow. This implies nonlocal dynamics and temporal coherence and is in accord with studies using analyzed winds in the stratosphere [Waugh and Plumb, 1994].

Figure 2 shows the corresponding results for the 4000 $\mathrm{K}$ isentropic surface (approximately $70-\mathrm{km}$ altitude), which is chosen to be representative of the middle mesosphere. In contrast to Figure 1, all four panels of Figure 2 differ significantly from one another, demonstrating that at this isentropic level the contour evolution is determined to a great extent by the high-frequency and small-scale components of the flow. The reference cal- culation (Figure 2a) shows plenty of small-scale structure, with none of the smooth elongation of contours that characterizes Figure 1. (Repeating the calculation using winds archived every 15 min produces essentially the same result.) The 18-hour sampling (Figure $2 \mathrm{~b}$ ) is clearly underresolved, leading to spuriously extended meridional displacements that reflect unrealistically long temporal persistence of the meridional flow. The existence of local dynamics is demonstrated by the sensitivity of the calculation to spatial truncation, as evidenced by the notable difference between Figures $2 \mathrm{a}$ and $2 \mathrm{c}$. If the mesospheric wind field is truncated in both space and time so as to be large-scale and slowly evolving, then the resulting contour evolution is forced to be spectrally nonlocal and temporally coherent and accordingly looks very "stratospheric" (Figure $2 \mathrm{~d}$ ). This illustrates that one can obtain something that 
looks like chaotic advection but which is an artifact of using a wind field that is artificially smooth in space and time (as mesospheric analyses presumably would be, if they existed).

The sensitivity of the above calculation to truncation of the wind field implies that Figure 2a cannot be considered fully representative of the real mesosphere in the way that Figure 1a can be considered fully representative of the real stratosphere. In particular, it seems quite clear that if CMAM were run at higher resolution, then the tracer evolution produced by that wind field would differ significantly from that produced by its T32 truncation. This suggests that results of off-line trajectory calculations using mesospheric winds are intrinsically resolution dependent and must be interpreted with care.

The increased spatial irregularity of the wind field between the stratosphere and mesosphere is clearly manifested in instantaneous snapshots of the zonal wind, shown in Figure 3 . At $1000 \mathrm{~K}(35 \mathrm{~km})$ there is a wavetwo "organizing structure" within a strong westerly flow in the Northern Hemisphere and very smooth wind isolines. At $2000 \mathrm{~K}(50 \mathrm{~km})$ this structure is still very much in evidence, but there is increased small-scale irregularity. By $4000 \mathrm{~K}(70 \mathrm{~km})$ the large-scale organizing structure associated with the stratospheric surf zone has disappeared and the wind field is very irregular; the mean is now much smaller than the deviations, even in the polar night jet.

In order to better characterize the small-scale structure of the wind field, kinetic energy spectra are shown in Figure 4. These spectra are computed as by Koshyk et al. [1999a] (except on isentropic surfaces) and are shown as a function of spherical harmonic index $n$, which represents the total horizontal wavenumber $k$ on the sphere (and will be used in place of $k$ throughout this section). Looking first at the total energy, the energy at $1000 \mathrm{~K}$ is dominantly in planetary scales, with a spectral tail somewhat steeper than a $n^{-3}$ power law (shown for comparison in the top right corner of each panel). At $4000 \mathrm{~K}$, by contrast, there is a shallower tail for $n>10$. (The scaling is roughly $n^{-1}$, though
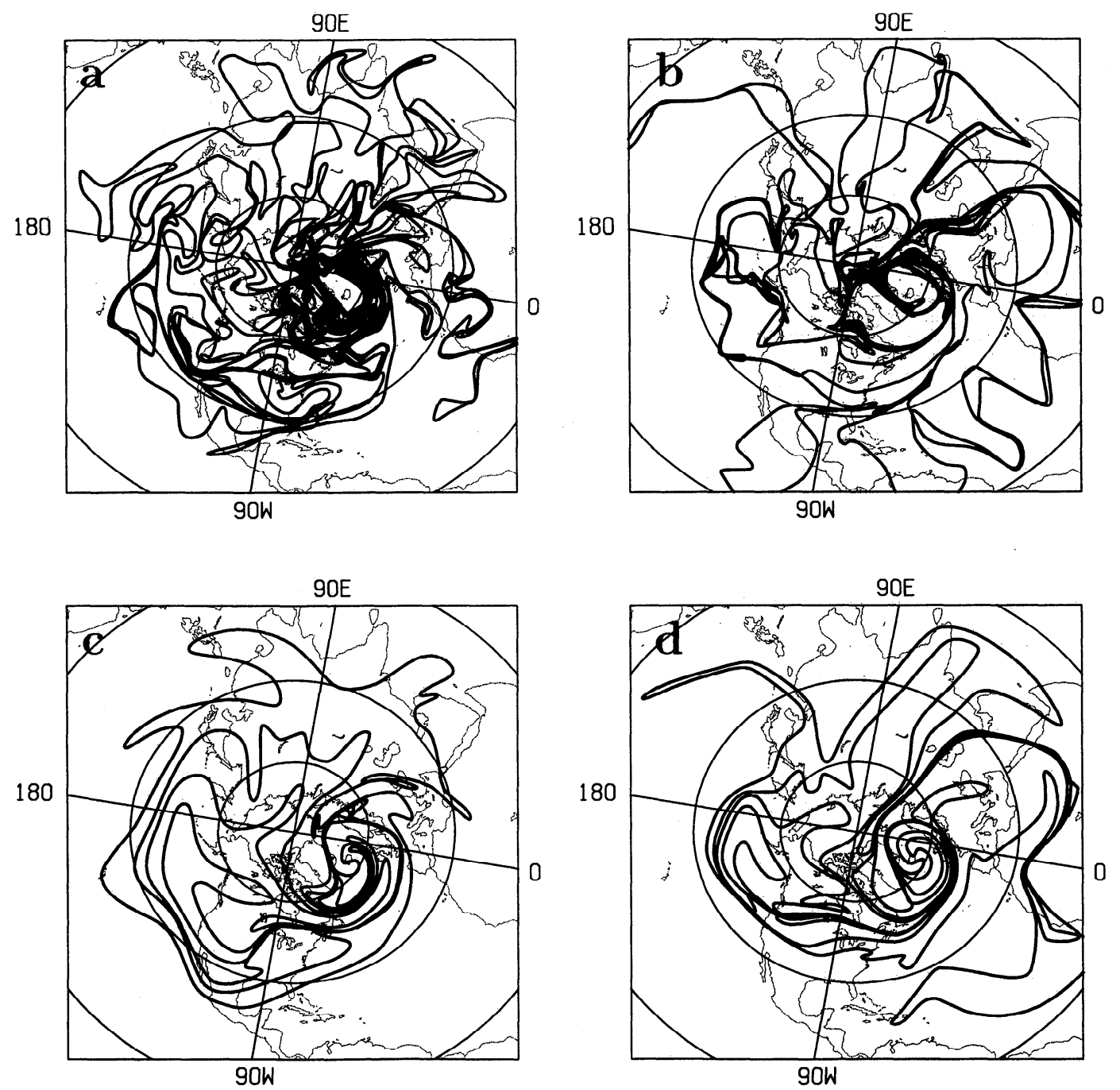

Figure 2. As Figure 1 but for the $4000 \mathrm{~K}$ isentropic surface. 

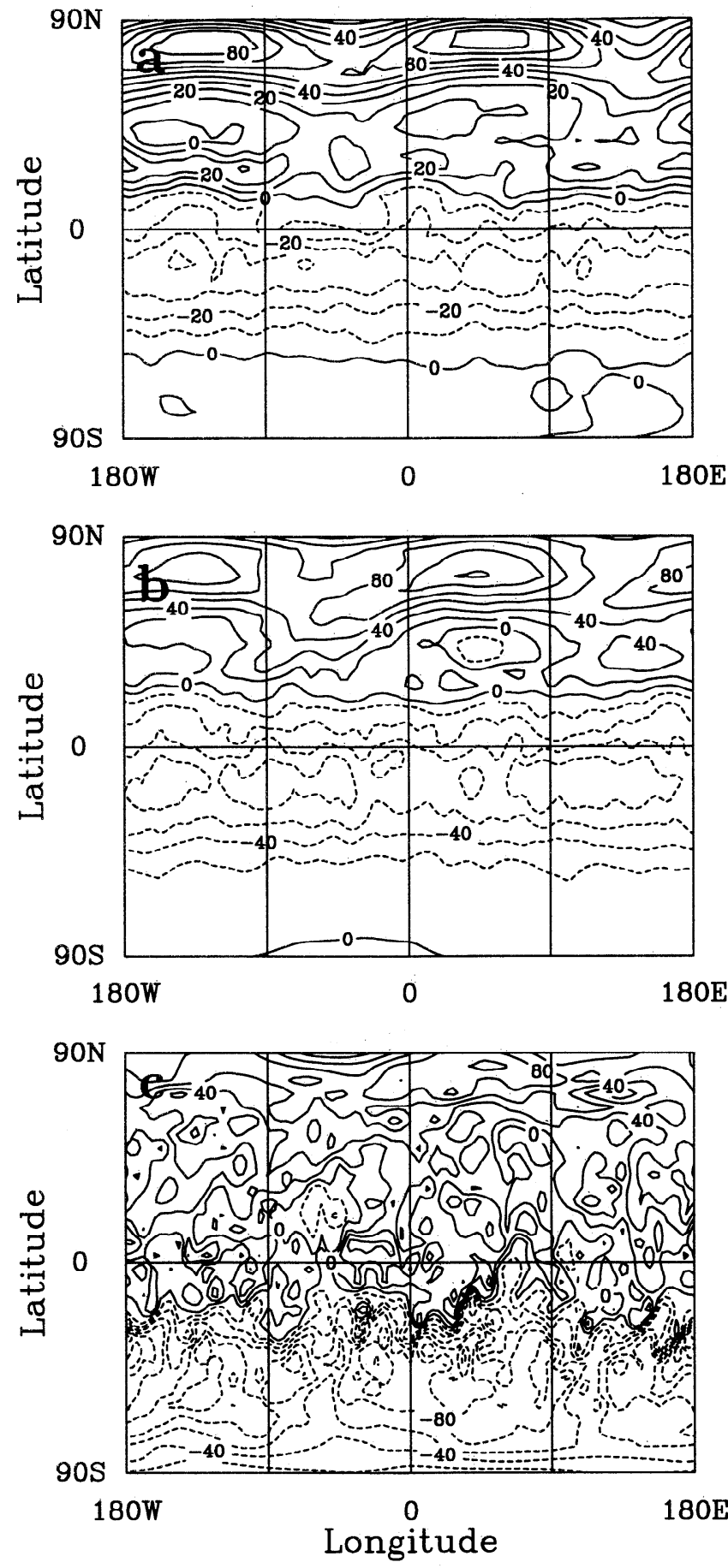

Figure 3. Snapshots of the horizontal wind field at the beginning of the advection period referred to in Figure 1 on the (a) $1000 \mathrm{~K}$, (b) $2000 \mathrm{~K}$, and (c) $4000 \mathrm{~K}$ isentropic surfaces. Contour intervals are $10 \mathrm{~m} \mathrm{~s}^{-1}$ in Figure $3 \mathrm{a}$ and $20 \mathrm{~m} \mathrm{~s}^{-1}$ in Figures $3 \mathrm{~b}$ and $3 \mathrm{c}$.

clearly one cannot put too much faith in any particular fit.) Examination of the rotational and divergent contributions to the spectrum reveals that this shallower tail is associated with an unbalanced component to the flow (divergent component comparable to rotational), which grows exponentially with altitude and eventually overwhelms the balanced component (divergent component much smaller than rotational) at the smaller scales. The "granular" nature of the instantaneous zonal wind at $4000 \mathrm{~K}$ seen in Figure $3 \mathrm{c}$ is thus understood to be the result of strong gravity wave activity at this altitude.

Because the unbalanced component of the flow has a shallower spectral slope than the balanced component, in the real atmosphere there must be a kink in the total kinetic energy spectrum at sufficiently high $n$. This kink is observed in the troposphere and lower stratosphere at about $n=100$ [Nastrom and Gage, 1985], which is well beyond the resolution of CMAM. (It has been reproduced in a recent high-resolution run of the GFDL SKYHI model [Koshyk et al., 1999b].) As the altitude increases, however, the kink migrates to smaller wavenumbers and cventually appears within the range of scales resolved by CMAM. Thus the character of the total kinetic energy spectrum in CMAM at $4000 \mathrm{~K}$ is quite generic. Exactly where the kink occurs is model dependent, since the strength of the gravity wave field in MAGCMs varies between models [Koshyk et al., 1999a]: a consequence of different spatial resolutions, gravity wave forcing mechanisms, and strength of numerical diffusion.

The steep kinetic energy spectrum that characterizes the stratosphere corresponds to nonlocal dynamics, with a single tracer timescale (and so is at least potentially consistent with chaotic advection), while the shallow spectrum that characterizes the mesosphere corresponds to local dynamics, with a scale-dependent tracer timescale. The phenomenological theory of Bennett [1984] and Babiano et al. [1985] therefore accounts quite well for the fact that the contour advection calculations are nonlocal (Figure 1) and local (Figure 2), respectively, in the two regions.

A limitation of the expression (1) is that it takes no account of spatial coherence or temporal irregularity. Chaotic advection requires not only nonlocal dynamics but also a suitablc "organizing structure" for transport and mixing, which is a generalization of the heteroclinic structure associated with time periodic chaotic advection [Ngan and Shepherd, 1999a, b]. Although some information can be gleaned from instantaneous snapshots of the velocity field, as in Figure 3 , it is essential to characterize the effect of the velocity field on the Lagrangian evolution. Contour advection calculations are not ideal for this purpose, since all particles are constrained to lie along single contours, and the implied tracer "field" therefore looks smoother than it really is. Thus domain-filling particle advection calculations were performed.

The results after 30 days are shown in Figure 5 for $1000 \mathrm{~K}$ and $4000 \mathrm{~K}$. The $1000 \mathrm{~K}$ panel shows a clear wave-two structure with two cats eyes; this is the signature of a breaking planetary wave two. The coherence of this structure over 30 days is remarkable. The $4000 \mathrm{~K}$ panel is very different: outside of the polar vortex, one sees no evidence of large-scale structure and 

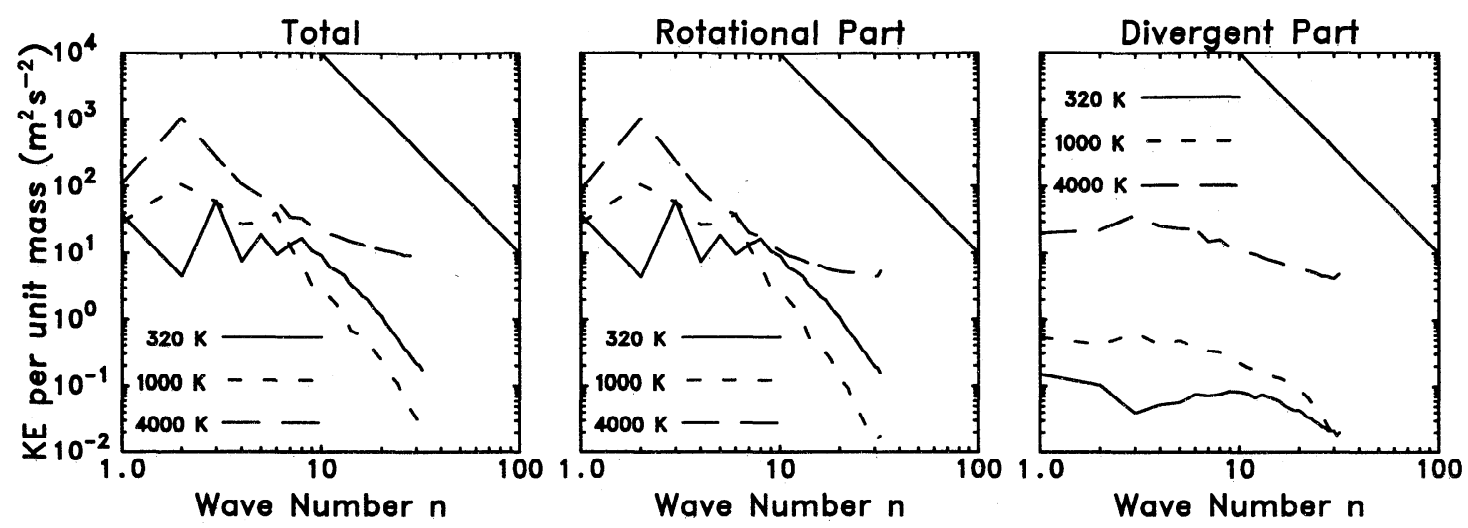

Figure 4. Monthly averaged kinetic energy spectrum per unit mass versus total horizontal wavenumber calculated from January Canadian Middle Atmosphere Model data. The total spectrum is shown in the left panel. Kinetic energy per unit mass associated with the rotational part of the flow alone (middle panel) and with the divergent part alone (right panel) are also shown. Data are plotted for three representative isentropic levels in each panel. The reference line in the upper right-hand corner of each panel has a slope of -3 .

(a)

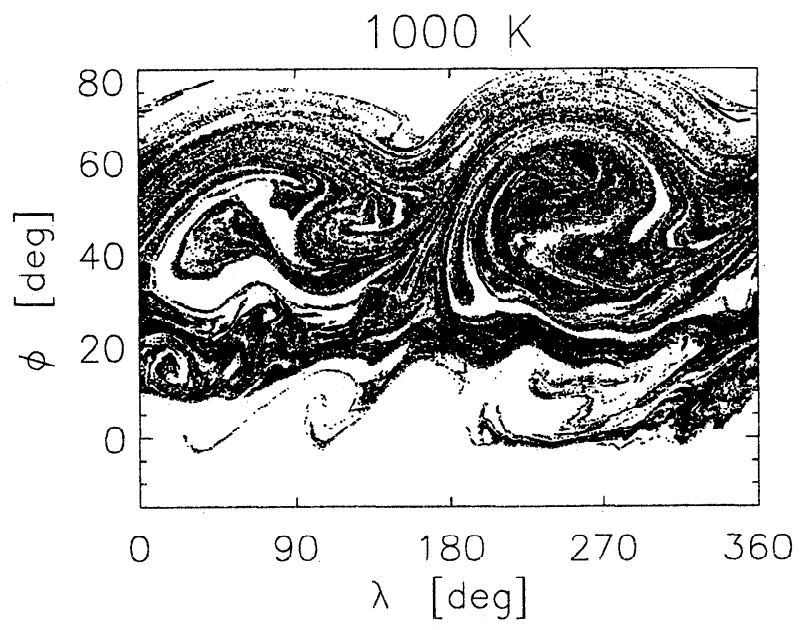

(b)

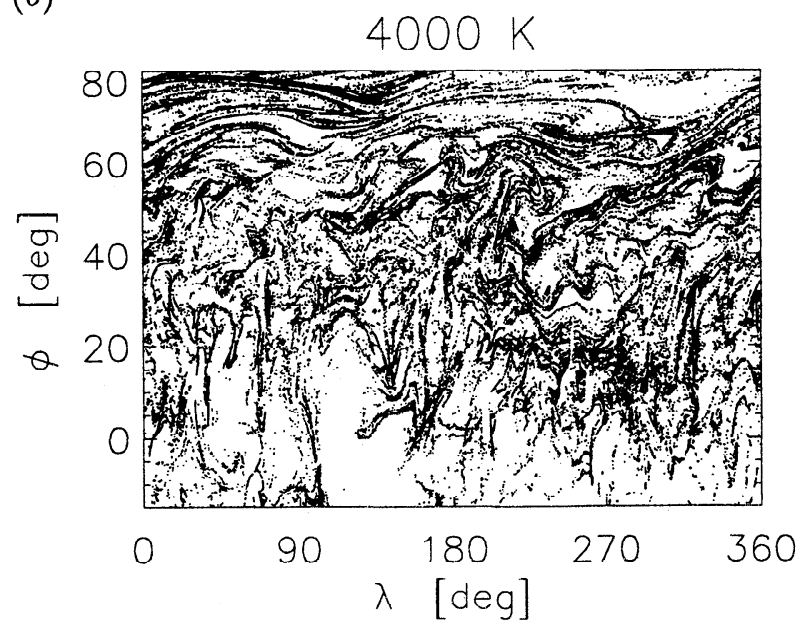

Figure 5. Tracer fields after 30 days: (a) $1000 \mathrm{~K}$; (b) $4000 \mathrm{~K}$. The initial distribution consists of approximately 250,000 points between $10^{\circ} \mathrm{N}$ and $70^{\circ} \mathrm{N}$. more of a diffusive, "fingering"--type structure. (Diffusion is, of course, an extreme case of local dynamics, where tracer evolution is determined by small-scale motion.) When the calculation at $4000 \mathrm{~K}$ is performed by using a T10, 18-hour truncation of the wind field (not shown), though the features are consistent with largescale advection, there is no particular evidence of an organizing structure such as one sees in the stratospheric case. Thus quite apart from the irregularity produced by gravity wave activity, the mesospheric flow appears to lack sufficient spatial organization to produce chaotic advection.

In order to quantify the temporal persistence of the strain field, temporal autocorrelations of velocity derivatives are examined. (Relative dispersion of particles is determined not by the velocity field, but by its derivatives, as in (1).) For a time series $u_{i}(i=1, \ldots, N)$, the autocorrelation $r(j)$ at lag $j$ is defined by

$$
r(j)=\frac{\frac{1}{N-j} \sum_{i=1}^{N-j}\left(u_{i}-\bar{u}\right)\left(u_{i+j}-\bar{u}\right)}{\frac{1}{N} \sum_{i=1}^{N}\left(u_{i}-\bar{u}\right)^{2}},
$$

where $\bar{u}=\frac{1}{N} \sum_{i=1}^{N} u_{i}$. Figure 6 compares the temporal autocorrelations of $u_{y}$, the meridional derivative of the zonal wind, for the same ensemblc of initial conditions used in Figure 5: the points are either advected by the flow (giving the Lagrangian correlation) or held at their initial location (giving the Eulerian correlation). At $1000 \mathrm{~K}$ (Figure 6a), the Lagrangian correlation decays quasi-exponentially and is essentially zero by about 3 days, whereas the Eulerian correlation decreases much more slowly and has not vanished even after 15 days. (The fact that the Lagrangian correlation drifts away from zero over long times reflects the nonstationarity of the mean flow.) Thus there is a finite Lagrangian correlation time but an undetermined (and certainly longer) Eulerian correlation time, implying that the Lagrangian 

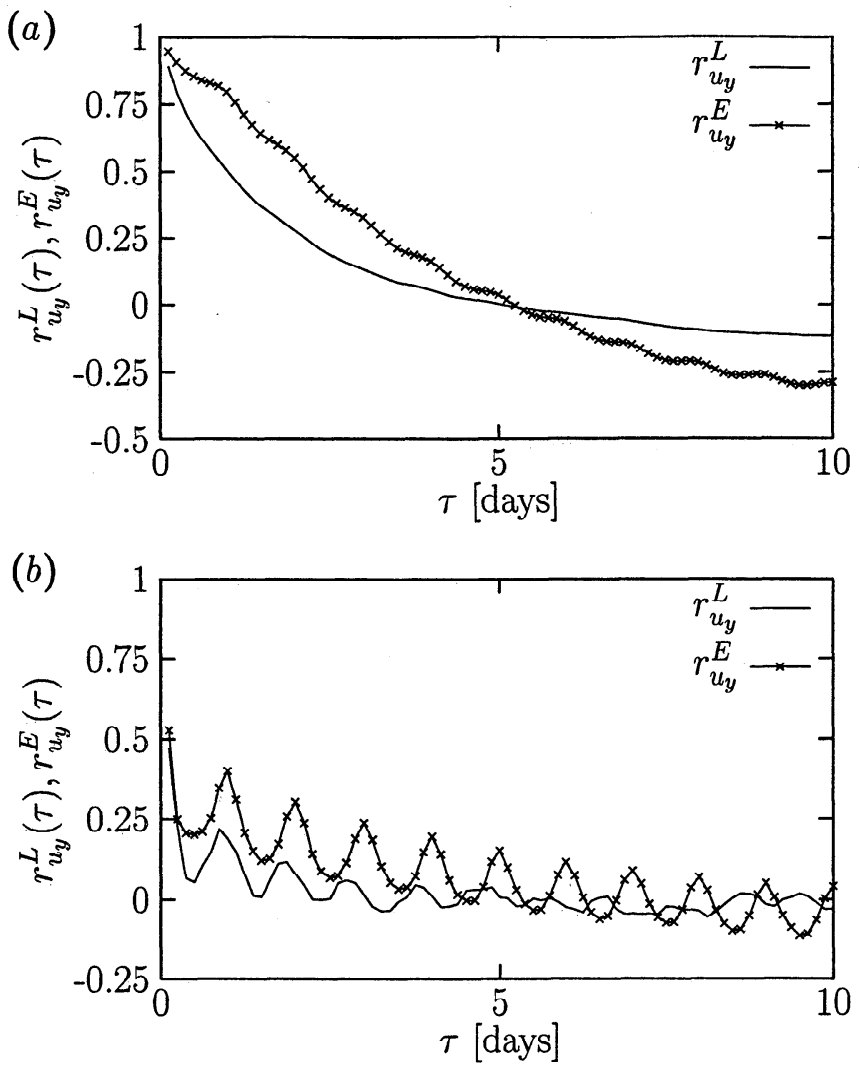

Figure 6. Eulerian and Lagrangian autocorrelations of $u_{y}$ : (a) $1000 \mathrm{~K}$; (b) $4000 \mathrm{~K}$. The Eulerian autocorrelations, $r_{u_{y}}^{E}$, are computed by using wind data between $10^{\circ} \mathrm{N}$ and $70^{\circ} \mathrm{N}$. The Lagrangian autocorrelations, $r_{u_{y}}^{L}$, are computed by using approximately 10,000 points initially lying between $10^{\circ} \mathrm{N}$ and $70^{\circ} \mathrm{N}$.

evolution is chaotic while the Eulerian flow is quasiregular: the defining characteristic of chaotic advection. Note that a Lagrangian correlation timescale of several days is comparable to inverse stretching rates found by using analyzed winds in the stratosphere [Schoeberl and Newman, 1995].

Figure $6 \mathrm{~b}$ shows the temporal autocorrelations at $4000 \mathrm{~K}$. In this case, both Eulerian and Lagrangian correlations drop sharply within 6 hours and then decay more slowly. Both correlations exhibit a clear diurnal oscillation, indicating the presence of a tide, but its overall amplitude is small. Evidently, there is no sharp distinction between the Lagrangian and Eulerian correlations at $4000 \mathrm{~K}$, with the exception of the greater persistence of the tidal oscillation visible in the latter; both correlations decay very rapidly. This is consistent with the hypothesis that chaotic advection is not applicable to the mesosphere.

The nonstationarity of the mean zonal flow during the integration period compromises, to some extent, the autocorrelation statistics of $u_{y}$ (the autocorrelations do not decay to zero as they should for a stationary random process). Thus the temporal autocorrelation of $v_{x}$, the zonal derivative of the meridional wind, was also examined, since the meridional wind has a much weaker zonal mean component; the results are shown in Figure 7. At $1000 \mathrm{~K}$, the Lagrangian correlation decays to zero by about 2 days and remains close to zero thereafter, with very little of the long-time "drift" seen in Figure 6. Fitting the data points to the function $a \exp (-t / \tau) \cos (\omega t)+(1-a)$ yields a correlation decay time $\tau$ of 0.5 day for the Lagrangian correlation $r^{L}$ and 2 days for the Eulerian correlation $r^{E}$. At $4000 \mathrm{~K}$, the same fit yields a correlation decay time of about 0.1 day in both cases. Thus the statistics of $v_{x}$ confirm the conclusions obtained from those of $u_{y}$ and exhibit less of a drift due to nonstationarity. The vertical dependence of the Lagrangian autocorrelation of $v_{x}$ is highlighted in Figure 8. It is quite evident that the correlation timescale is much shorter at $4000 \mathrm{~K}$ than at $1000 \mathrm{~K}$.

Another characteristic of chaotic advection is the large-scale spatial inhomogeneity of mixing at finite times. This phenomenon can be studied by examining the PDF of finite time Liapunov exponents over a chaotic region. Finite time Liapunov exponents measure the average stretching rate experienced by a fluid element over the time interval in question. Specifically, a Liapunov exponent $\lambda_{i}$ is defined at each $t$ by

$$
\lambda_{i}=\lim _{\delta x_{i}(0) \rightarrow 0}\left\{\frac{1}{t} \ln \frac{\delta x_{i}(t)}{\delta x_{i}(0)}\right\},
$$
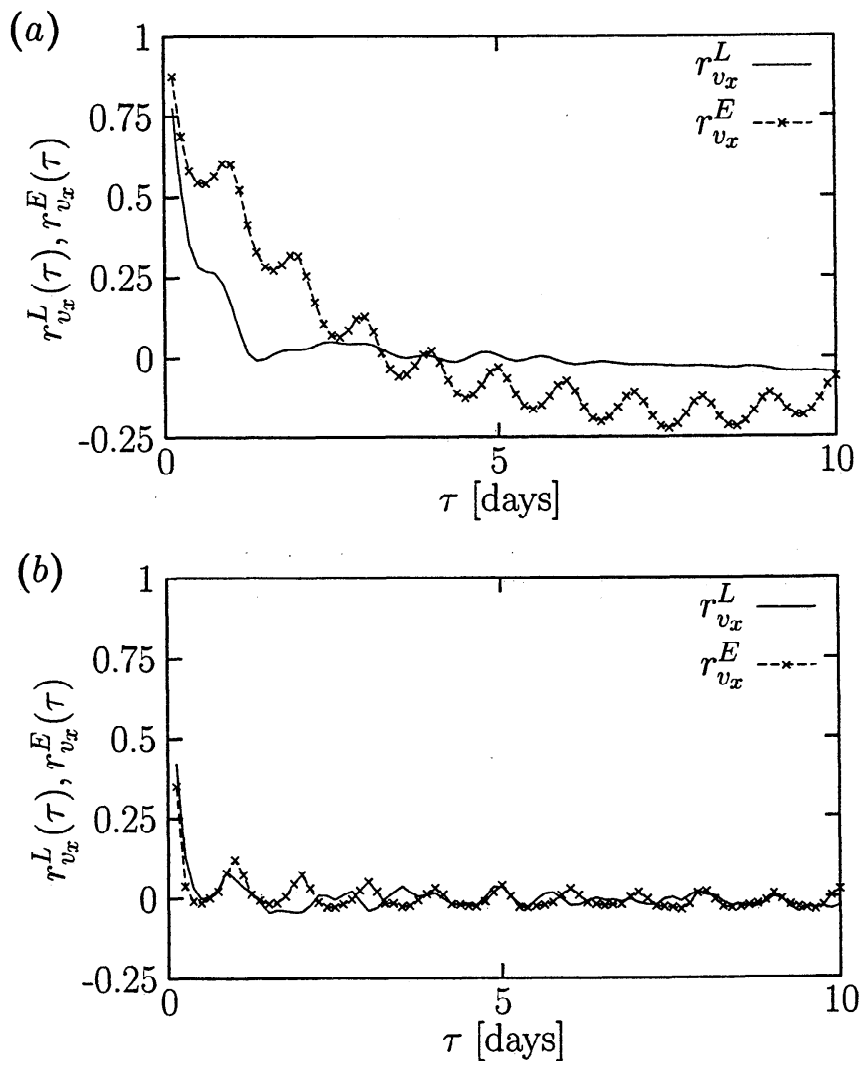

Figure 7. Eulerian and Lagrangian autocorrelations of $v_{x}$ : (a) $1000 \mathrm{~K}$; (b) $4000 \mathrm{~K}$. The initial distributions are the same as those in Figure 6. 


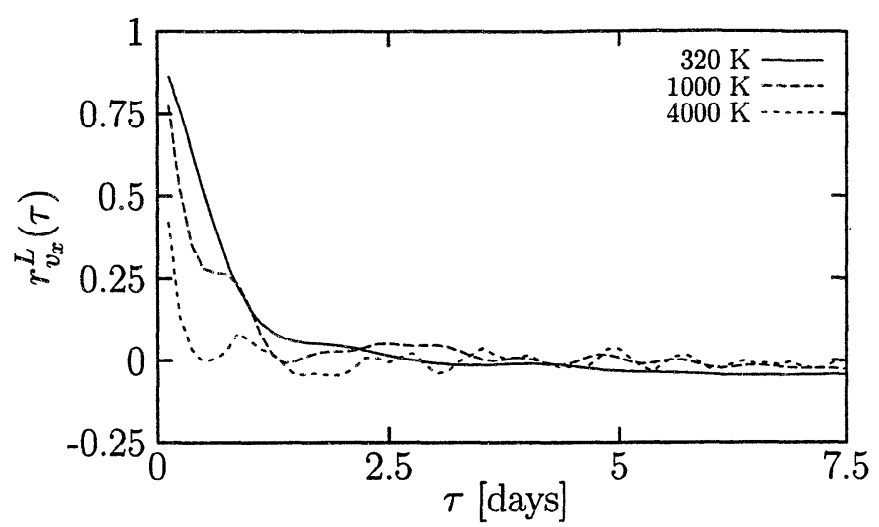

Figure 8. Lagrangian autocorrelation $r_{v_{x}}^{L}(\tau)$ for the $320 \mathrm{~K}, 1000 \mathrm{~K}$, and $4000 \mathrm{~K}$ isentropic surfaces. The calculations are as in Figure 6.

where $\delta x_{i}(t)$ is the separation of two trajectories, under the linearized dynamics, displaced by a distance $\delta x_{i}(0)$ at $t=0$. (In practice, the $\lambda_{i}$ are calculated from the linearized flow operator.) The PDF is constructed from the $\lambda_{i}$ (i.e., it is a normalized histogram). For stationary statistics and a single chaotic region, one expects the PDF to approach a Gaussian in the long-time limit, as fluid elements randomly sample the entire chaotic region, narrowing to a delta function (the largest "true" Liapunov exponent), with perhaps some zeroes associated with fluid elements trapped in nonchaotic regions. Roughly speaking, the broader the PDF, at any given time, the more inhomogeneous is the mixing, and the more Gaussian the PDF, the more ergodic is the sampling of the chaotic region.

Since the stretching statistics of the model wind fields are not stationary, the behavior of the PDFs may not conform exactly to the expectations described above. It also follows that better statistics cannot be obtained by extending the integrations. This problem is related to the drift in the autocorrelations described earlier.

Figure 9 shows the PDFs of Liapunov exponents for the particle ensemble used in Figure 5, for $1000 \mathrm{~K}$ and $4000 \mathrm{~K}$. The different curves show the sensitivity to using different subsets of the wind data (as in Figures 1 and 2). At $1000 \mathrm{~K}$, there is fairly close agreement between all calculations, which is consistent with nonlocal dynamics. The PDFs are strongly skewed to large stretching rates, with a long tail, which suggests that most of the stretching comes from rare, intense events; even after 30 days, mixing at $1000 \mathrm{~K}$ is highly inhomogeneous. (This is consistent with the organizing structure for mixing seen in Figure 5.) At $4000 \mathrm{~K}$, the PDFs are quite sensitive to filtering of the velocity field. The PDF for T10, 3-hour truncation is displaced toward smaller Liapunov exponents, confirming that most of the stretching in the unfiltered velocity field comes from its smaller-scale components. This is consistent with the picture of local dynamics. The PDF at full spatial and temporal resolution (T32, 3-hour truncation) is rea- sonably Gaussian, suggesting greater ergodicity than at $1000 \mathrm{~K}$, although there remains a non-Gaussian tail.

The PDF for T32, 18-hour truncation is displaced toward larger Liapunov exponents. This is presumably an artifact of the unrealistically long persistence of the filtered velocity field, as was seen in Figure 2b. As was discussed by Chertkov et al. [1995], Liapunov exponents are increased when the correlation time of the strain is increased. (Although their study is restricted to largescale random strain, we expect this result to apply more generally.) This effect can also be seen in the vertical dependence of the PDFs as shown in Figure 10. It is seen that the mean Liapunov exponent is smallest at $1000 \mathrm{~K}$ and the PDF is most skewed. As the altitude increases, the mean Liapunov exponent first increases and then decreases (maximizing at $3000 \mathrm{~K}$ ). This decrease, despite the increase in kinetic energy (and therefore the amplitude of the velocity derivatives) at all scales (Figure 4), is once more associated with a decrease in the persistence of the velocity field as the altitude increases (as shown in Figure 8).

The presence of significant gravity wave activity is analogous to noise in the velocity field. Ngan and Shepherd $[1999 \mathrm{a}, \mathrm{b}]$ considered the effect of externally im-
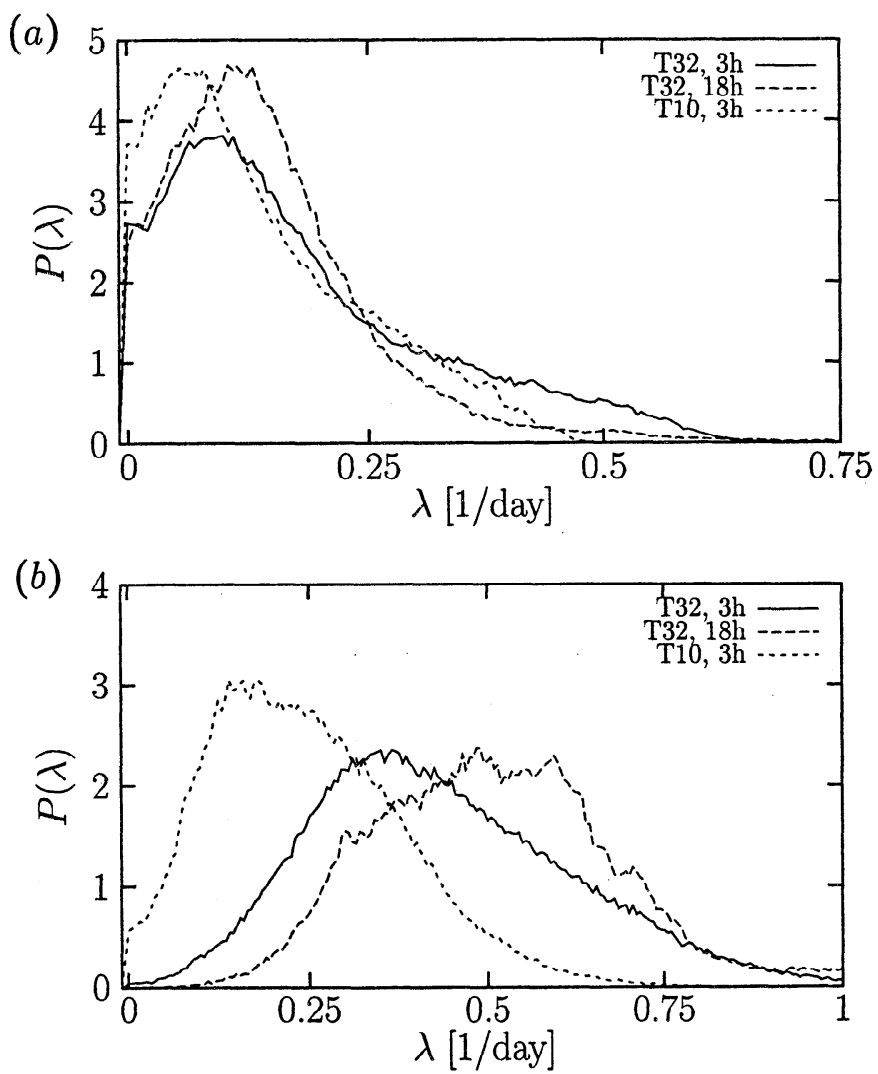

Figure 9. $P(\lambda)$ after a 30 -day integration using various truncations of the wind field: (a) $1000 \mathrm{~K}$; (b) $4000 \mathrm{~K}$. The initial distributions are the same as those in Figure 5. Particles north of $80^{\circ} \mathrm{N}$ are excluded from the calculation. 


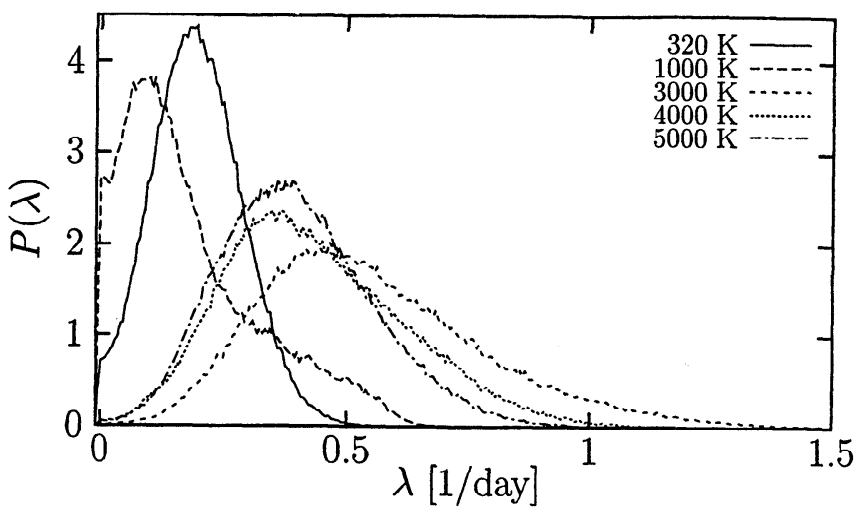

Figure 10. $P(\lambda)$ at 30 days for various isentropic surfaces. The calculations are as in Figure 9.

posed noise on a breaking planetary wave in shallow water flow and showed that for a sufficiently strong stochastic perturbation, chaotic advection fails to apply. That result may therefore be seen to anticipate the present one. Of course, in that study the noise was completely artificial; here it is generated internally and self-consistently by the model in the form of gravity waves.

Up to this point, this study has focused on the contrast between the (middle) stratosphere and mesosphere. It is also instructive to look at isentropic surfaces that intersect the extratropical tropopause. At those altitudes, synoptic-scale disturbances are active and the energy spectrum is not band limited to the planetary scales, as it is higher up. Observations of upper tropospheric winds show an approximately $n^{-3}$ power law scaling of the kinetic energy spectrum [Boer and Shepherd, 1983] consistent with two-dimensional turbulence theory, and as was noted in the introduction, this power law is exactly on the borderline between spectrally local and nonlocal dynamics. Thus although there have been a number of Lagrangian transport studies in this region [e.g., Pierrehumbert and Yang, 1993; Appenzeller et al., 1996; Bithell and Gray, 1997], it is not a priori evident that the conditions of nonlocal dynamics, let alone chaotic advection, apply.
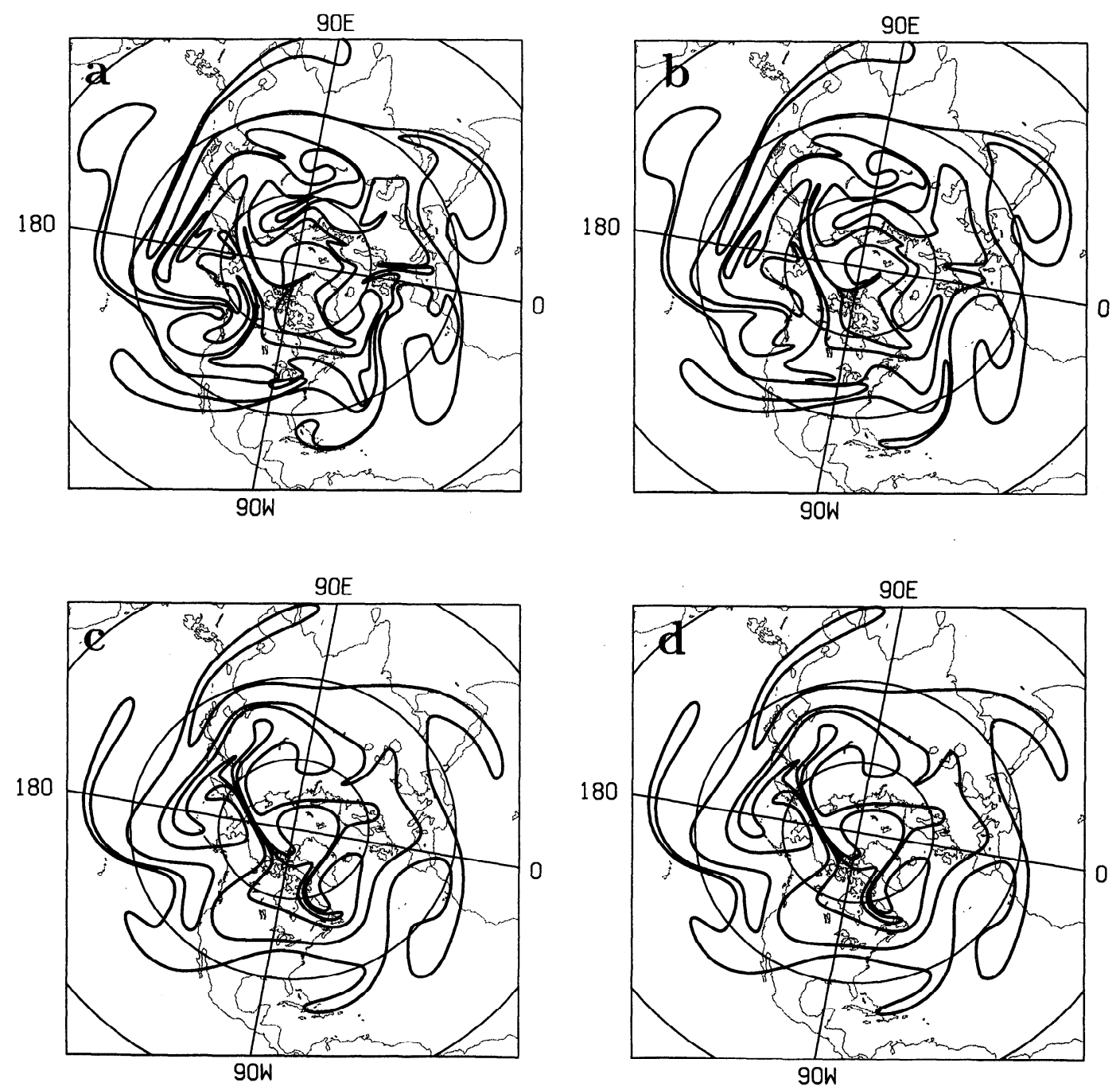

Figure 11. Same as Figure 1 but for the $320 \mathrm{~K}$ isentropic surface. 
The $320 \mathrm{~K}$ kinetic energy spectra are shown in Figure 4 and are seen to reproduce these observed features. In particular, the total energy spectrum indicates much more active synoptic scales than at $1000 \mathrm{~K}$ and relatively weaker planetary scales, clear evidence of Charney-Drazin filtering between $320 \mathrm{~K}$ and $1000 \mathrm{~K}$. The spectrum is consistent with $n^{-3}$ from about $n=$ $10-20$, which is outside the dissipation range of the model. Contour advection calculations using these $320 \mathrm{~K}$ winds are shown in Figure 11. There is clearly more evidence of synoptic-scale structure than in Figure 1, including two cats eye structures over the eastern Atlantic and the Middle East; yet the generally filamentary character of the contours is still evident. There are no qualitative differences between the various panels, although the T10 truncations (Figures 11c and 11d) clearly miss some of the deformation.

In order to determine whether chaotic advection provides a reasonable approximation to adiabatic transport across the extratropical tropopause, Eulerian and Lagrangian temporal autocorrelations of $v_{x}$ at $320 \mathrm{~K}$ are considered in Figure 12. The Lagrangian correlation decays by about 2-3 days, as in the middle stratosphere (Figure 7a). The Eulerian correlation decays on a similar timescale, although there is a bit of persistent overshoot, and the correlation has not really vanished. Nevertheless, the Eulerian and Lagrangian correlations appear to be much more similar than they are in Figure $7 \mathrm{a}$. Indeed fitting the data points to the function $a \exp (-t / \tau) \cos (\omega t)+(1-a)$ yields a decay time $\tau$ of about 1 day for the Lagrangian correlation and 0.7 day for the Eulerian correlation. The similarity between these two values suggests that chaotic advection may be inapplicable in this region.

The PDFs of stretching rates, shown in Figure 13, are evidently much more symmetric than at $1000 \mathrm{~K}$. Since the Lagrangian correlation time is similar (see Figure 8 ), this suggests that the greater ergodicity that is implied at $320 \mathrm{~K}$ must come from greater spatial homogeneity of the velocity field, which is consistent with the dominance of synoptic-scale rather than planetary-

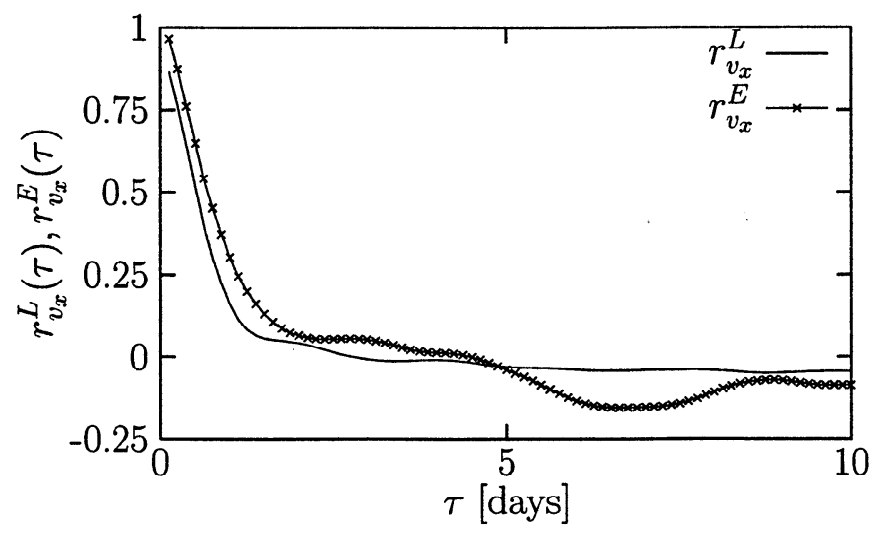

Figure 12. Eulerian and Lagrangian autocorrelations at $320 \mathrm{~K}$. The calculations are as in Figure 6.

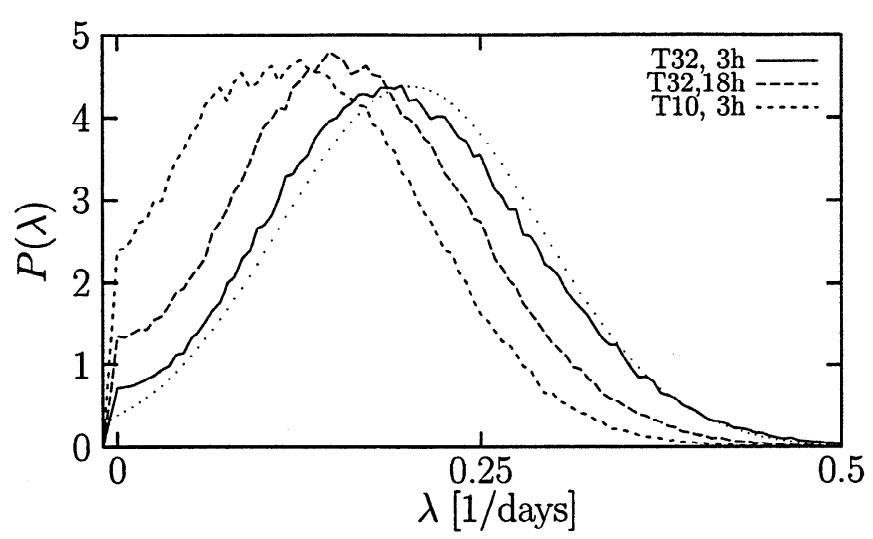

Figure 13. $P(\lambda)$ after a 30 -day integration using various truncations of the $320 \mathrm{~K}$ winds. The normal distribution for the T32, 3-hour case is plotted as a dotted line. The calculations are as in Figure 9.

scale structures. This by itself does not exclude the possibility of chaotic advection, but it does appear to exclude the existence of a global-scale organizing structure. This suggests that a horizontally homogeneous random strain description of mixing may be appropriate in this region. However, such a description requires a single timescale for mixing, and the general arguments from (1) would suggest that such a timescale does not exist, because the dynamics are weakly nonlocal. This is consistent with the fact that the PDF computed by using T10 winds provides a significant underestimate of the mean Liapunov exponent computed by using T32 winds, unlike the stratospheric case.

Thus from these diagnostics it is difficult to justify the relevance of chaotic advection to mixing across the extratropical tropopause. Nevertheless, the situation is much more benign in this respect in the vicinity of the tropopause than in the mesosphere. The flow is still essentially balanced, and there is some degree of band limiting in frequency. Thus Lagrangian calculations are still reasonably meaningful, if not completely well defined as in the stratosphere. (One expects a weak scale dependence, since the spectral slope is marginal.) Certainly, there is nothing like the strong spatio-temporal irregularity that is seen in the mesosphere.

\section{Summary and Discussion}

The nature of adiabatic Lagrangian motion in the extratropical wintertime middle atmosphere has been considered by using winds from the Canadian Middle Atmosphere Model to provide a surrogate atmosphere. The $1000 \mathrm{~K}$ and $4000 \mathrm{~K}$ isentropic surfaces have been studied in detail, as being representative of the middle stratosphere and middle mesosphere, respectively. Consistent with other studies using analyzed winds, it is found that adiabatic motion in the stratosphere is predominantly determined by the large-scale lowfrequency flow. This situation arises in the stratosphere 
because Charney-Drazin filtering acts to remove all but the planetary scales, while gravity waves have yet to reach significant amplitudes. It follows that the Lagrangian evolution is chaotic while the Eulerian evolution is quasi-regular, which is the defining characteristic of chaotic advection.

In the mesosphere, on the other hand, there is a strong gravity wave component in the model which introduces significant temporal and spatial irregularity into the wind field. This irregularity is reflected in the kinetic energy spectra, which exhibit a shallow tail (approximately a $k^{-1}$ power law scaling), the flow being largely unbalanced in this range (divergent component comparable to rotational). Under these circumstances, the conditions required for chaotic advection do not apply: Lagrangian and Eulerian correlation timescales are similar (and very short, on the scale of hours), and tracer evolution is spectrally local. Thus quasi-horizontal tracer advection in the mesosphere is controlled primarily by the gravity wave spectrum.

Figure 14 shows the Eulerian and Lagrangian correlation times $\tau_{E}$ and $\tau_{L}$ for the velocity derivative $v_{x}$ as a function of potential temperature $\theta$. Presented in this fashion, the results highlight the very special nature of the middle stratosphere: only in this region is $\tau_{E}$ clearly long in comparison with $\tau_{L}$. There is an abrupt drop in $\tau_{E}$ between the middle and upper stratosphere, and above the stratopause the values of $\tau_{E}$ and $\tau_{L}$ are quite similar. An interesting point is that $\tau_{E}$ and $\tau_{L}$ both decrease dramatically with altitude in the mesosphere (this was noted earlier in the discussion concerning Figure 10). Using ground-based lidar data, Hauchecorne et al. [1991] found a dramatic drop in the correlation of observed 7-day mean temperatures above $65 \mathrm{~km}$. Al-

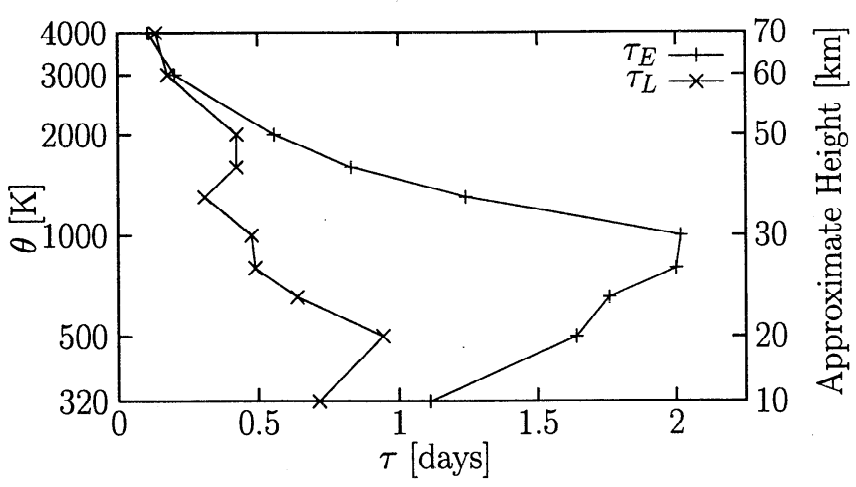

Figure 14. Eulerian and Lagrangian correlation times versus potential temperature (and approximate height); $\tau_{E}$ and $\tau_{L}$ are computed by fitting the 30-day time series of $r_{v_{x}}^{E}$ and $r_{v_{x}}^{L}$, respectively, to the function $f(t)=$ $a \exp (-t / \tau) \cos (\omega t)+(1-a)$, where $a$ and $\omega$ are parameters, using the Levenberg-Marquadt method [Press et al., 1992]. In the fitting the different data points are weighted equally (i.e., it is assumed that the errors are identical). Similar results are obtained from the 10-day and 20-day time series. though this is a different statistic for a different field, it may be a reflection of the same underlying phenomenon.

Figure 14 also shows a noticeable drop in $\tau_{E}$ between the middle and lowermost stratosphere. In the latter region, the ratio of correlation times is much closer to unity; both correlation times decrease as the altitude decreases, presumably reflecting the increasing role of synoptic-scale disturbances.

It may be objected that since the observed stratospheric wind field also exhibits a shallow tail to its kinetic energy spectrum, with approximately a $k^{-5 / 3}$ power law scaling for wavelengths smaller than $1000 \mathrm{~km}$ [Bacmeister et al., 1996], the conclusions derived for the mesosphere should also apply to the stratosphere at sufficiently small scales. While this may be true in principle, it appears not to be so in practice. According to (1) it is the total stretching rates that matter, and local dynamics will only become operative sufficiently far into the shallow spectrum range, once the r.m.s. strain from the small scales begins to dominate over that from the large scales. In the lower stratosphere, the shallow tail only appears on the mesoscale [Nastrom and Gage, 1985 ] and should therefore make a negligible contribution to the total stretching rates. This is reflected in the fact that the filamentary nature of lower stratospheric tracer fields, with their charactcristic edgelike structure, persists with a $k^{-2}$ scaling throughout the mesoscale range down to the smallest horizontal scales resolved in the aircraft data, of about $0.5 \mathrm{~km}$ [Bacmeister et al., 1996]. This structure reflects the dominance of the large-scale velocity field [Ngan and Shepherd, 1997]. Thus the scale at which local dynamics becomes operative would be so small that tracer advection would be fully three dimensional on short timescales.

The spectral locality of tracer advection in the mesosphere implies that contour or particle advection calculations in this region are resolution dependent and must be interpreted with care. In particular, studies performed by using coarse-grained analyzed winds (if they existed) would not adequately represent the transport and mixing properties of the mesosphere. Indeed, mesospheric calculations might give a false impression of chaotic advection, because of the artificial spatiotemporal filtering that is built into most analysis products. This is in contrast with the stratosphere, where temporal coherence of the velocity field together with spectral nonlocality (which is precisely the setting for chaotic advection) allows for meaningful trajectory calculations. Danilin et al. [2000] have developed a "trajectory hunting" method for combining noncoincident chemical measurements; the present results show that the effectiveness of this method may be limited to the stratosphere.

How much spatio-temporal resolution is then required in the mesosphere in order to properly represent mixing? The answer to this question depends on the problem. If the objective is to make a deterministic calculation of a particular parcel trajectory, then the situation 
is essentially hopeless; one would have to resolve the velocity field all the way down to the dissipation range. If, on the other hand, the objective is to represent mixing in a statistical sense, the situation is much more promising: the evolution of tracers appears to be significantly more diffusive in the mesosphere than in the stratosphere, when viewed on the large scales, and a coarse truncation together with diffusion may be sufficient. A proper characterization of mixing and transport in the mesosphere is thus an interesting avenue for future work.

The severe temporal and spatial irregularity of the mesospheric wind field has important implications for wind measurements in this region. Clearly, the sampling requirements are likely to be far more stringent than those in the stratosphere, and the prospects for data assimilation are likely to be daunting: without significant spatial or temporal coherence in the wind field, data gaps may be difficult to cover.

Acknowledgments. This study was supported by the Canadian Middle Atmosphere Modelling project, through grants from the Natural Sciences and Engineering Research Council of Canada and the Climate Research Network of the Atmospheric Environment Service. Work by K.N. at the University of Chicago was supported by the National Oceanic and Atmospheric Administration under grant NA56GP0436 and by the National Science Foundation under grant ATM-9505190. The authors are grateful to the reviewers for several useful suggestions.

\section{References}

Andrews, D. G., J. R. Holton, and C. A. Leovy, Middle Atmosphere Dynamics, 489 pp., Academic, San Diego, Calif., 1987.

Appenzeller, C., H. C. Davies, and W. A. Norton, Fragmentation of stratospheric intrusions, J. Geophys. Res., 101, 1435-1456, 1996.

Babiano, A., C. Basdevant, and R. Sadourny, Structure functions and dispersion laws in two-dimensional turbulence, J. Atmos. Sci., 42, 941-949, 1985.

Bacmeister, J. T., S. D. Eckermann, P. A. Newman, L. Lait, K. R. Chan, M. Loewenstein, M. H. Proffitt, and B. L. Gary, Stratospheric horizontal wavenumber spectra of winds, potential temperature, and atmospheric tracers observed by high-altitude aircraft, J. Geophys. Res., 101, 9441-9470, 1996.

Balsley, B. B., and D. A. Carter, The spectrum of atmospheric velocity fluctuations at $8 \mathrm{~km}$ and $86 \mathrm{~km}$, Geophys. Res. Lett., 9, 465-468, 1982.

Bartello, P., Using low-resolution winds to deduce fine structure in tracers, Atmos. Ocean, 38, in press, 2000.

Beagley, S. R., J. de Grandpré, J. N. Koshyk, N. A. McFarlane, and T. G. Shepherd, Radiative-dynamical climatology of the first-generation Canadian middle atmosphere model, Atmos. Ocean, 35, 293-331, 1997.

Bennett, A. F., Relative dispersion: Local and nonlocal dynamics, J. Atmos. Sci., 41, 1881-1886, 1984.

Bithell, M., and L. J. Gray, Contour lengthening rates near the tropopause, Geophys. Res. Lett., 24, 2721-2724, 1997.

Boer, G. J., and T. G. Shepherd, Large-scale two-dimensional turbulence in the atmosphere, J. Atmos. Sci., 40, 164$184,1983$.

Chertkov, M., G. Falkovich, I. Kolokolov, and I. Lebedev,
Statistics of a passive scalar advected by a large-scale twodimensional velocity field: analytic solution, Phys. Rev. E Stat. Phys. Plasmas Fluids Relat. Interdiscip. Top., 51, 5609-5627, 1995.

Danilin, M. Y., M. L. Santee, J. M. Rodriguez, M. K. W. Ko, J. M. Mergenthaler, J. B. Kumer, A. Tabazadeh, and N. J. Livesey, Trajectory hunting: A case study of rapid chlorine activation in December 1992 as seen by UARS, J. Geophys. Res., 105, 4003-4018, 2000.

Dritschel, D. G., Contour dynamics and contour surgery: Numerical algorithms for extended, high-resolution modelling of vortex dynamics in two-dimensional, inviscid, incompressible flows, Comput. Phys. Rep., 10, 78-146, 1989.

Geist, K., U. Parlitz, and W. Lauterborn, Comparison of different methods for computing Lyapunov exponents, Prog. Theor. Phys., 83, 875-893, 1990.

Gibson-Wilde, D. E., R. A. Vincent, C. Souprayen, S. Godin, A. Hertzog, and S. D. Eckermann, Dual lidar observations of mesoscale fluctuations of ozone and horizontal winds, Geophys. Res. Lett., 24, 1627-1630, 1997.

Hauchecorne, A., M.-L. Chanin, and P. Keckhut, Climatology and trends of the middle atmosphere temperature (33-87 km) as seen by Rayleigh lidar over the south of France, J. Geophys. Res., 96, 15,297-15,309, 1991.

Hayashi, Y., D. G. Golder, J. D. Mahlman, and S. Miyahara, The effect of horizontal resolution on gravity waves simulated by the GFDL SKYHI general circulation model, Pure Appl. Geophys., 130, 421-443, 1989.

Koshyk, J. N., B. A. Boville, K. Hamilton, E. Manzini, and K. Shibata, The kinetic energy spectrum of horizontal motions in middle atmosphere models, J. Geophys. Res., 104, 27,177-27,190, 1999a.

Koshyk, J. N., K. Hamilton, and J. D. Mahlman, Simulation of the $k^{-5 / 3}$ mesoscale spectral regime in the GFDL SKYHI general circulation model, Geophys. Res. Lett., 26, 843-846, 1999b.

McIntyre, M. E., and T. N. Palmer, Breaking planetary waves in the stratosphere, Nature, 305, 593-600, 1983.

Nastrom, G. D., and K. S. Gage, A climatology of atmospheric wavenumber spertra of wind and temperature observed by commercial aircraft, J. Atmos. Sci., 42, 950960, 1985.

Newman, P. A., and M. R. Schoeberl, A reinterpretation of the data from the NASA stratosphere-troposphere exchange project, Geophys. Res. Lett., 22, 2501-2504, 1995.

Ngan, K., and T. G. Shepherd, Comments on some recent measurements of anomalously steep $\mathrm{N}_{2} \mathrm{O}$ and $\mathrm{O}_{3}$ tracer spectra in the stratospheric surf zone, J. Geophys. Res., 102, 24,001-24,004, 1997.

Ngan, K., and T. G. Shepherd, A closer look at chaotic advection in the stratosphere, part I, Geometric structure, J. Atmos. Sci., 56, 4134-4152, 1999a.

Ngan, K., and T. G. Shepherd, A closer look at chaotic advection in the stratosphere, part II, Statistical diagnostics, J. Atmos, Sci., 56, 4153-4166, 1999b.

Norton, W. A., Breaking Rossby waves in a model stratosphere diagnosed by a vortex-following coordinate system and a technique for advecting material contours, J. Atmos. Sci., 51, 654-673, 1994.

Orsolini, Y. J., On the formation of ozone laminae at the edge of the Arctic polar vortex, Q. J. R. Meteorol. Soc., 121, 1923-1941, 1995.

Pierrehumbert, R. T., and H. Yang, Global chaotic mixing on isentropic surfaces, J. Atmos. Sci., 50, 2462-2480, 1993.

Press, W. H., S. A. Teukolsky, W. T. Vetterling, and B. P. 
Flannery, Numerical Recipes in C: The Art of Scientific Computing, 2nd ed., 994 pp., Cambridge Univ. Press, New York, 1992.

Schoeberl, M. R., and P. A. Newman, A multiple-level trajectory analysis of vortex filaments, J. Geophys. Res., $100,25,801-25,815,1995$.

Strahan, S. E., and J. D. Mahlman, Evaluation of the SKYHI general circulation model using aircraft $\mathrm{N}_{2} \mathrm{O}$ measurements, 2, Tracer variability and diabatic meridional circulation, J. Geophys. Res., 99, 10,319-10,332, 1994.

Swanson, K. L., and R. T. Pierrehumbert, Lower-tropospheric heat transport in the Pacific storm track, J. Atmos. Sci., 54, 1533-1543, 1997.

Thuburn, J., and D. G. H. Tan, A parameterization of mixdown time for atmospheric chemicals, J. Geophys. Res., 102, 13,037-13,049, 1997.

von Bremen, H. F., F. E. Udwadia, and W. Proskurowski, An efficient QR based method for the computation of Lyapunov exponents, Physica D, 101, 1-16, 1997.

Waugh, D. W., and R. A. Plumb, Contour advection with surgery: A technique for investigating fine-scale structure in tracer transport, J. Atmos. Sci., 51, 530-540, 1994.
Waugh, D. W., et al., Transport out of the lower stratospheric Arctic vortex by Rossby wave breaking, J. Geophys. Res., 99, 1071-1088, 1994.

Whiteway, J. A., and A. I. Carswell, Lidar observations of gravity wave activity in the upper stratosphere over Toronto, J. Geophys. Res., 100, 14,113-14,124, 1995.

Wilson, R., M. L. Chanin, and A. Hauchecorne, Gravity waves in the middle atmosphere observed by Rayleigh lidar, 2, Climatology, J. Geophys. Res., 96, 5169-5183, 1991.

J. N. Koshyk, K. Ngan, and T. G. Shepherd, Department of Physics, University of Toronto, 60 St. George Street, Toronto, Ontario M5S 1A7, Canada. (koshyk@atmosp. physics.utoronto.ca; keith@atmosp.physics.utoronto.ca; tgs@atmosp.physics.utoronto.ca)

(Received July 1, 1999; revised February 3, 2000; accepted February 9, 2000.) 